Meta

Journal des tradlucteurs

Translators' Journal

\title{
Pour une théorie de l'essence de la traduction
}

\section{Vassilis Koutsivitis}

Volume 38, numéro 3, septembre 1993

URI : https://id.erudit.org/iderudit/003348ar

DOI : https://doi.org/10.7202/003348ar

Aller au sommaire du numéro

Éditeur(s)

Les Presses de l'Université de Montréal

ISSN

0026-0452 (imprimé)

1492-1421 (numérique)

Découvrir la revue

Citer cet article

Koutsivitis, V. (1993). Pour une théorie de l'essence de la traduction. Meta, 38(3), 468-472. https://doi.org/10.7202/003348ar d'utilisation que vous pouvez consulter en ligne.

https://apropos.erudit.org/fr/usagers/politique-dutilisation/ 


\title{
POUR UNE THÉORIE DE L'ESSENCE DE LA TRADUCTION
}

\author{
VASSILIS KOUTSIVITIS \\ Université Ionienne, Corfou, Grèce
}

\section{A. LES THÉORIES DE LA TRADUCTION}

Les théories de la traduction ont traversé trois phases jusqu'à présent:

a) la phase prélinguistique (jusqu'au début du XXe siècle): approche philologique et philosophique de traducteurs qui essaient d'approfondir leur travail ;

b) la phase linguistique (jusqu'aux années 60): analyse systématique et factuelle du phénomène traductif au niveau de la langue;

c) la phase métalinguistique (les vingt dernières années): tentative de synthèse des approches précédentes de la part de traductologues avec l'apport de disciplines nouvelles (sémiologie, théorie de la communication, textologie,...).

Cette tentative de synthèse, si nous nous limitons à l'époque contemporaine, fut également la réponse des praticiens-théoriciens (Nida, Seleskovitch, Ladmiral) à la thèse des linguistes (Fedorov, Mounin, Catford) concernant la traduction en tant que phénomène linguistique et à l'antithèse des empiristes (Cary, Steiner, Meschonnic) postulant le caractère subjectif de l'activité traduisante.

Aujourd'hui, nous pouvons dire qu'on commence à s'approcher d'une théorie unique et universelle de la traduction. Certes, à cause de la complexité du phénomène traductif, il y a des chercheurs qui privilégient soit les éléments linguistiques, soit les contenus cognitifs, soit les aspects anthropologiques, soit les nuances littéraires, et ainsi de suite. Cependant, d'ores et déjà, une majorité toujours croissante de traductologues provenant de différents horizons, indépendamment des terminologies qu'ils utilisent et des classifications auxquelles ils aboutissent, s'accordent fondamentalement pour voir dans la traduction, malgré ses multiples facettes, un phénomène unique; ce phénomène 1) se résume à la transposition de l'essence d'un texte qui contient des éléments sémantiques et stylistiques, 2) se situe au niveau de l'expression matérialisée, 3) vise à la communication et 4) se comprend par l'interprétation.

Il est compréhensible qu'au début ce phénomène unique ait êté disséqué par des chercheurs embarrassés par sa complexité. Il est également explicable que d'autres sciences du langage, ou de l'homme en général, aient tenté d'analyser certains aspects de ce phénomène, en considérant qu'elles l'élucident dans son intégralité. Néanmoins, la nouvelle science de la traduction, la traductologie, en délimitant son objet, en élaborant ses propres méthodes, s'est constituée progressivement en une discipline scientifique à part entière.

\section{B. L'ESSENCE DE LA TRADUCTION}

La traduction est une nouvelle rédaction d'un texte existant. Une rédaction en langue $\mathrm{X}$ d'un texte établi en langue $\mathrm{Y}$, basée sur les connaissances thématiques et les aptitudes linguistiques adéquates. L'élaboration de ce nouveau texte se décompose en trois phases: compréhension du texte de départ, rédaction du texte d'arrivée, évaluation. 
Ainsi, la traduction englobe en même temps trois états correspondant respectivement à ces trois phases: un processus, une pratique, un produit.

Si on approfondit l'analyse du processus traductif, on constate qu'on ne traduit pas des mots, même pas des phrases, mais des textes. Mais quand on traduit un texte, on ne traduit pas le texte dans sa totalité, car le transfert total d'un texte signifierait sa répétition et non pas sa traduction. On traduit l'essence du texte. La traduction consiste donc en le transfert de l'essence d'un texte d'une langue à une autre.

L'essence d'un texte a un caractère holistique et monistique. C'est grâce à ce caractère de l'essence du texte qu'on peut y fonder une approche globale de la traduction. Elle comporte des aspects notionnels, émotionnels et formels. Elle contient des éléments explicites et implicites. Dans un sens large, l'essence d'un texte englobe son auteur, son contexte et sa finalité.

La langue est le vecteur mais pas le maître de la traduction; elle lui est indispensable mais elle n'est pas déterminante. Il s'agit de deux ensembles qui s'entrecoupent, qui se recouvrent par endroits, mais qui ne s'identifient d'aucune manière. Il n'existe pas d'effort plus vain et plus dangereux, en traductologie, que de vouloir examiner la traduction au niveau de la langue.

Si la traduction est toujours possible, elle n'est jamais totale, parce que l'équation entre la pensée et le verbe n'existe pas, parce que la traduction totale - phonétique, graphique, grammaticale, lexicale, stylistique - équivaudrait à la répétition du texte original et non pas à une nouvelle rédaction.

Ainsi, la traduction peut atteindre différents degrés : équivalence, correspondance, égalité, même identité (toujours partielle, c'est le cas des emprunts dans un texte). Elle peut, alors, changer de nom et au lieu de «traduction» s'appeler interprétation, paraphrase, adaptation, imitation.

De toute façon, nous ne pouvons discerner que trois états purs du processus traductif:

a) le transcodage, soit la correspondance d'un à un ;

b) la traduction, soit l'équivalence d'un à plusieurs; et

c) l'interprétation, soit la création, libre de toute correspondance ou équivalence préétablie.

Dans tous les cas le problème central de la traduction est la découverte de l'intention communicative de l'auteur de l'original et la reproduction de son impact.

\section{L'UNITÉ ET L'UNICITÉ DE LA TRADUCTION}

La traduction est un phénomène unique, se caractérisant par une unité interne. Malgré ses expressions diverses elle est toujours régie par quelques principes fondamentaux communs.

La traduction est un acte de parole et non pas un fait de langue. Le traducteur, en étant bilingue, opère comme un double unilingue qui, après avoir saisi l'essence d'un texte établi dans une langue, la rend dans une autre.

L'essence d'un texte est la réalité psychique et mentale que l'émetteur du message veut et essaie de communiquer, et que le récepteur saisit grâce à son support sémantique et stylistique, mais aussi grâce au contexte général.

Dans toute traduction, nous discernons, d'une part, des éléments codifiés ou codifiables ayant des équivalences fixes en langue-cible et, d'autre part, des énoncés originaux que le traducteur reformule en les interprétant librement. L'objectif de la traduction est finalement l'identité du sens et l'équivalence de la forme, la fidélité à l'essence du texte, qui est la synthèse de son contenu et de sa forme. 
En parlant de liberté et de fidélité en traduction, il faut préciser qu'il ne s'agit pas d'une antinomie mais d'une symbiose. Une traduction réussie est libre (recréation interprétative) et fidèle (transfert du message), une traduction ratée est «libre» (paraphrase) et «fidèle» (transposition linguistique).

Un autre paramètre important et commun dans toute traduction est son caractère social. Le traducteur joue obligatoirement un rôle de médiateur dans la chaîne de communication qui le met en exergue, mais parfois l'accable aussi.

Il adopte, consciemment ou inconsciemment, une attitude par rapport au message ainsi qu'à son émetteur et à son récepteur et ceci influe sur l'opération traduisante.

Enfin, la traduction en tant que (nouvelle) rédaction, et non pas transcodage, dispose d'un outil de travail unique, à savoir, la langue-cible. D'où la nécessité d'orienter davantage l'effort vers la langue d'arrivée que vers la langue de départ ou le domaine thématique. Cela s'applique à une situation idéale où le traducteur dispose du bagage nécessaire à ces trois nivaux. Dans le cas contraire (souvent rencontré dans la pratique), il est évident que les handicaps doivent être corrigés en priorité.

Le traducteur a toujours été, et il l'est de plus en plus, un défenseur, un promoteur et un créateur de sa langue au même titre que l'écrivain. Dans quelques langues, notamment de petite diffusion, et dans quelques domaines, dits de pointe, l'apport et la responsabilité du traducteur sont beaucoup plus importants que ceux de l'écrivain.

Le fait que toute traduction obéisse à des principes fondamentaux communs ne doit pas nous conduire à minimiser l'importance des différences et des particularités des matérialisations multiformes de ce phénomène.

\section{LA DUALITÉ ET LA PLURALITÉ DE LA TRADUCTION}

L'existence de deux pôles fondamentaux, original-traduction, prédispose à un état dualiste qui se manifeste de multiples façons.

Il y a d'abord la scission en traduction écrite et traduction orale (interprétation), et à un second niveau la division de la première en traduction de textes littéraires et traduction de textes pragmatiques.

La première scission, qui a lieu à partir de la distinction entre langue parlée et langue écrite sans se réduire uniquement à cela, même si les principes de base restent les mêmes, cela n'empêche pas que l'interprétation constitue, par son naturel, sa spontanéité, son déroulement in vivo, un poste privilégié d'observation du processus, tandis que la traduction écrite, par sa permanence, sa rigueur, son caractère sophistiqué, se prête mieux à l'analyse du produit traductif.

Quant à la deuxième division, même si la fonction expressive domine dans les textes littéraires et la fonction informative dans les textes pragmatiques, la synthèse de la forme et du contenu caractérise les deux. Même si l'accent est mis, chez les premiers, sur l'aspect émotif et chez les seconds, sur l'aspect cognitif, il s'agit de deux aspects fondamentaux de la même entité, de l'essence du texte.

Mais, de l'autre côté, il est certain qu'en traduction littéraire, il faut privilégier le rythme et en traduction pragmatique, le terme. La traduction poétique et la traduction technique représentent les deux extrêmes du processus et du produit traductifs en termes de liberté/fidélité, interprétation/transcodage, impossible/possible; cependant, il s'agit toujours de deux extrêmes du même continuum.

Deux autres catégorisations importantes du phénomène traductif sont les caractéristiques et les exigences différentes de la traduction synchronique et diachronique, ainsi que de la traduction interlinguistique et intralinguistique. 
De toutes les contradictions coexistant et en interaction dans le processus traductif, celle qui, à mon avis, est caractéristique de ce phénomène est le fait que toute traduction est, à des degrés différents, un amalgame d'opérations standardisées et non standardisées.

D'une part, les correspondances répétitives transcodables, de l'autre, les équivalences inédites contextuelles. D'un côté, les formules, les termes, le transcodage, de l'autre, le texte libre, l'interprétation. Le traducteur se meut continuellement à l'instar d'une pendule, entre ces deux extrêmes, à la recherche du mouvement juste, du bon rythme.

\section{E. PANTRADUCTISME ET MÉTATRADUCTISME}

La place de plus en plus importante de la traduction dans le monde contemporain a donné naissance au «pantraductisme», c'est-à-dire à une approche qui tend à examiner tout phénomène comme un phénomène de traduction. Les activités les plus fondamentales et les plus caractéristiques de l'homme - parler, écouter, lire, comprendre, communiquer dans une ou plusieurs langues, synchroniquement ou diachroniquement — peuvent être envisagées, et le sont toujours à un certain degré, comme une traduction.

La convergence à tous les niveaux, phénomène marquant de la seconde moitié du $\mathrm{XX}^{\mathrm{e}}$ siècle, est aussi en partie le résultat de la traduction. La convergence linguistique par la traduction correspond à la convergence des cultures, des systèmes politiques et économiques, ainsi que des rapports humains, grâce à l'internationalisation sans précédent et toujours croissante des relations humaines, grâce aussi au développement des médias et des autres moyens de communication.

Dans ses visées de pantraductisme, la traductologie fait appel à une multitude de disciplines auxiliaires qui, de leur côté, peuvent aussi la revendiquer comme auxiliaire à elles : la terminologie, la lexicologie, la linguistique générale et contrastative, la sémiotique, la sémantique, l'étude des langues de spécialité, la didactique des langues, la linguistique computationnelle, l'ethnologie, la sociologie, la neurolinguistique, la science de la communication, la textologie, la philologie, la critique littéraire et la littérature comparée, l'analyse du discours, la stylistique générale et comparative...

L'impossibilité pour la traduction de tout englober, de tout résoudre, suscite la dynamique et crée les fondements pour son dépassement. D'un côté, avec la promotion de langues universelles, naturelles ou artificielles, et(ou) par l'étude de langues étrangères. De l'autre, par la contestation de ses droits et par la fixation des limites arbitraires à son intervention.

Le mouvement pantraductif provoque ainsi une réaction métatraductive, à savoir une tendance à minimiser, voire anihiler la faisabilité et(ou) l'utilité de la traduction.

Mais la «menace» la plus grave et, en même temps, la plus optimiste qui pèse sur l'activité et la réflexion traductive, comme elle pèse sur la quasi-totalité des activités humaines au seuil du XXIe siècle, est son dépassement par une autre création humaine, l'informatique et la robotique. Il s'agit de la traduction automatique.

Les théories de la traduction contemporaines se sont développées presque parallèlement à l'évolution informatique. Cependant ce parallélisme n'était ni linéaire ni calme. la première rencontre avait provoqué le coup de foudre et un enthousiasme réciproque. Les premières difficultés avaient conduit à la rupture. La sagesse du temps a contribué à une relation plus réaliste et constructive.

L'informatisation ne remplace pas l'opération traduisante humaine. D'abord, elle l'assiste de plusieurs façons (traitement de texte, base de données terminologiques et documentaires, etc.). Ensuite, elle s'y substitue partiellement et imparfaitement. Cette substitution grandit continuellement tout en diminuant ses imperfections. 
Mais, même quand la machine pourra tout traduire, et parfaitement, il faudra encore des hommes pour construire ces machines.

En cet avenir proche ou lointain, la pratique traduisante ne sera plus la même qu'aujourd'hui, les théories de la traduction non plus. Mais les principes essentiels du phénomène traductif resteront les mêmes.

\section{RÉFÉRENCES}

Nous nous limitons à la mention des dix livres les plus représentatifs en matière de théorie de la traduction contemporaine :

BERMAN, A. (1984) : L'épreuve de l'étranger, Paris, Gallimard.

CARY, E. (1963) : Les grands traducteurs français, Genève, Librairie de l'Université Georg et Cic.

DELISLE, J. (1980): L'analyse du discours comme méthode de traduction, Ottawa, Éditions de l'Université d'Ottawa.

LADMIRAL, J.-R. (1979): Traduire : théorèmes pour la traduction, Paris, Payot.

MOUNIN, G. (1963): Les problèmes théoriques de la traduction, Paris, Gallimard.

NEWMARK, P. (1988) : A Texthook of Translation, London, Prentice Hall.

NIDA, E. A. et C. R. TABER (1982) : The Theory and Practice of Translation, Leiden, E. J. Brill.

SELESKOVITCH, D. et M. LEDERER (1984) : Interpréter pour traduire, Paris, Didier Érudition.

STEINER, G. (1975) : After Babel. Aspects of Language and Translation, London, Oxford University Press.

VINAY, J.-P. et J. DARBELNET (1958) : Stylistique comparée du français et de l'anglais, Paris, Didier.

ainsi que de la première tentative probante d'une synthèse critique :

LAROSE, R. (1989): Théories contemporaines de la traduction, Québec, Presses de l'Université du Québec. 